\title{
AN INDEPENDENT-FLOW-FIELD MODEL FOR A SDOF NONLINEAR STRUCTURAL SYSTEM, PART II: ANALYSIS OF COMPLEX RESPONSES
}

\author{
Huan Lin \\ e-mail: linh@engr.orst.edu \\ Solomon C.S. Yim \\ e-mail: solomon.yim@oregonstate.edu
}
Ocean Engineering Program, Department of Civil Engineering Oregon State University, OR 97331, U.S.A.

\begin{abstract}
Complex responses observed in an experimental, nonlinear, moored structural system subjected to nearly periodic wave excitations are examined and compared with the simulations of a newly proposed independent-flow-field (IFF) model in this paper. Variations in wave heights are approximated by additive random perturbations to the dominant periodic component. Simulations show good agreement with the experimental results in both time and frequency domains. Noise effects on the experimental results including bridging and transition phenomena are investigated and interpreted by comparing with the simulations of its deterministic counterpart. Possible causes of a chaotic-like experimental result as previously observed are also inferred.
\end{abstract}

Key Words: experimental, nonlinear, random perturbations, complex 


\section{Introduction}

A wealth of complex nonlinear response behavior, including chaos, of ocean structural systems under deterministic monochromatic wave excitations had been studied analytically and demonstrated via computer simulations [1-2]. Nonlinearities of the system resulted from a combination of geometrically nonlinear restoring force and (quadratic) drag force. In a deterministic setting, local and global stability analyses were conducted, and stability regions of various nonlinear responses were identified [1-2]. A fine bifurcation super-structure was found [3] and the sequential ordering indices of bifurcation implied the existence of higher order nonlinear responses (e.g., ultra-sub-harmonic and quasi-periodic) and possible routes to chaos. However, chaotic responses in full-scale engineering systems in the field were yet to be identified.

Small-scale experiments had been carried out to search the existence of chaotic responses. Under such easily controlled environments, experimental chaotic responses were found to exist [4]. To closely simulate field conditions, a medium-scale experimental study was performed for highly nonlinear ocean structures subjected to periodic and nearly periodic waves [5]. An intent of the experimental study was to search and identify nonlinear complex structural responses, even chaos in an environment closer to wave fields. Despite good agreements shown between numerical predictions and experimental results, there were experimental observations that could not be explained using only deterministic analysis procedures [6], such as an "unexpected" transition from (transient) harmonic to (steady-state) sub-harmonic response. The "unexpected" transition was attributed to (unaccounted for) variations in wave heights. Random perturbation components thus needed to be incorporated in the analytical models despite the fact that the physical structural system was designed to behave in a deterministic manner in the experiment [5]. Because of the random nature of wave fields, the model with perturbed periodic wave excitation could more closely simulate structural response behavior in the field.

Based on the experimental results, analytical theory and numerical results were developed to identify and analyze the effects of weak random noise on periodically driven 
nonlinear moored structures [7]. Analytical results indicated the capability of utilizing response probability density functions to depict complex responses including noisy chaos and rich nonlinear phenomena such as bifurcations and coexisting response attractors. A preliminary comparison and analysis of experimental results were also conducted [8]. Results of the experimental study demonstrated noise effects on responses in the time domain, and assessed possible existence of noisy chaos. As discussed in Part I of this study, in spite of relatively good predictions, the previously employed model was not able to fully capture the overall experimental response behavior. Neither super- nor sub-harmonic resonance was identified and captured in the simulated results. More detailed investigations of the experimental results were therefore suggested [8].

This study (Part II) focuses on the investigation of the response behavior of a moored structure subjected to nearly periodic waves. The variations of the wave amplitudes are taken into account by additive random perturbations. An independent-flow-field (IFF) model with nonlinear structure and nonlinear damping is employed to more closely describe the experimental response behavior. The IFF model is considered the most suitable for the experiment in the wave frequency range examined [9] and validated by extensive numerical, parametric studies in Part I. The model adopts an alternate form of Morison drag using independent flow field, and adds a quadratic term in the polynomial approximation to the restoring force. Good agreements between numerical predictions and experiment results, in both overall behavior and individual response trajectories under regular waves, have been shown in Part I. The objectives of this study are twofold: 1) to investigate underlying intricate bifurcation patterns near resonances, and 2) to interpret complex nonlinear phenomena observed, as in [8] and discussed in previous studies including perturbation effects on nonlinear responses and "possibly noisy chaotic" experimental results.

\section{System Considered}

The multi-point moored structural system considered in this study is formulated as a 
single-degree-of-freedom (SDOF) submerged rigid body, hydrodynamically damped and excited nonlinear oscillator with random perturbations. The experimental configurations are reported in detail by Yim et al [5], and a summary description of the model, including setup and test classifications, is presented in Part I.

\section{Equation of Motion}

The response behavior of a nearly periodically driven moored offshore structure can be modeled by the following equation [9]:

$M \ddot{x}+C_{S} \dot{x}+C_{D}^{\prime} \dot{x}|\dot{x}|+R(x)=F_{D}(u)+F_{I}(\dot{u}, \ddot{x})+\xi(t)$

where $x$ and $\dot{x}$ denote the surge displacement and velocity, respectively; $M$, mass of sphere; $R$, nonlinear restoring force; $C_{S}$, effective (linear) system damping coefficient $\left(=\zeta_{S} C_{c r} ; \zeta_{S}\right.$, damping ratio and $C_{c r}$, critical damping); $C_{D}^{\prime}$, hydrodynamic damping coefficient; $F_{D}$ and $F_{I}$, drag and inertial components of the exciting force, respectively. Note the addition of the band-limited random perturbation term $\xi(t)$ (cf. Eq.(1) in Part I) in the equation.

The restoring force (due to nonlinear geometric configuration), $R(x)$, is given by Eq.(2) in Part I, and the $90^{\circ}$ mooring configuration is again emphasized here. The exciting force consists of a Morison drag $\left(F_{D}\right)$ and an inertial component $\left(F_{I}\right), F_{D}=\frac{\rho}{2} C_{D} A_{P} u|u|$, and $F_{I}=\rho \forall\left(1+C_{A}\right) \frac{\partial u}{\partial t}-\rho \forall C_{A} \ddot{x}$, respectively, where $C_{D}$ is the hydrodynamic viscous drag coefficient; $C_{A}$, added mass coefficient; $A_{P}$, projected drag area; $\forall$, displaced volume; $\rho$, water density; and $u$, water particle velocity.

The stochastic excitation component is described by a band-limited noise perturbations, $\xi(t)$, incorporating all possible randomness in the wave field. The Rice noise (band-limited) representation is chosen here to provide a practical noise model [6]. The simulated structural response will be used to compare with experimental data in a later section. The perturbation component, $\xi(t)$, is given by 
$\xi(t) \cong \sum_{j=1}^{N} a_{j} \cos \left(\omega_{j} t-\varphi_{j}\right)$

where the $a_{\mathrm{j}}$ 's are amplitudes, $\omega_{\mathrm{j}}$ 's frequencies, and $\varphi_{\mathrm{j}}$ 's uniformly distributed independent random variables in the interval of $[0,2 \pi]$, respectively. In the experiment, monochromatic waves with random perturbations were generated based on given energy spectrum densities with designed noisy intensities.

One of the primary intents of the experiment was to investigate the effects of smallintensity perturbations on the characteristics of nonlinear responses. Each test was then performed sufficiently long to ensure that the transient response had been damped out. Data acquisition tests were initially performed over a range of frequencies and wave heights at which interesting nonlinear responses may be expected based on the deterministic testing data. In the deterministic tests, the model with the $90^{\circ}$ configuration (cf. Fig. 1 in Part I, b=0) subjected to 2

foot, 2 second waves exhibited sub-harmonic nonlinear behavior. The wave period of 2 seconds was selected for all tests, and the wave amplitudes varied within the range of $1.5-2.5 \mathrm{ft}$. The random noise variance was designed to be “small” ranging within 15\% of the total input energy.

Under the specified wave excitation conditions described above, a total of 9 tests were conducted and the model responses were recorded (Tests D4-13, see Yim et al [5] for detailed documentation). All the tests exhibited both harmonic and sub-harmonic characteristics in the response, and possible coexistence was implied. As indicated in the frequency domain, when the wave amplitude was increased, the strength of the sub-harmonic component increased. Moreover, analysis in the time domain showed oscillations between the two possible coexisting distinct response attractors, i.e., harmonic and sub-harmonic.

\section{Identification of Noise Intensity and System Parameters}

The presence of random and uncontrollable components in the wave excitation is incorporated in the analytical model as a band-limited noise as shown in Eq.(2). Experimental 
observations indicate that besides the designed (analytically specified) band-limited noise component, there exist additional, uncontrollable "random" perturbations due to imperfections in the wave generation and bounded wave field. The effects of these imperfections (caused by diffractions, reflections and return currents in the wave flume), together with the analytically specified noise intensity in the waves are identified as demonstrated by Yim and Lin [8].

Identification of system parameters $M, \mathrm{C}_{a}, C_{S}, C_{D}, C_{D}^{\prime}, k_{1}, k_{2}$ and $k_{3}$ (see Eqs.(1-2) here and Eqs.(2-3) in Part I) of the analytical model are conducted utilizing the model configurations, results of free vibration tests, initial estimates of a frequency technique, and extensive numerical simulations and comparisons as briefly described in Part I. The set of 'best' constant coefficients is identified and given by Table 1 in Part I.

\section{Deterministic Analysis}

Because of the "smallness" of the random perturbations, the deterministic analysis results can provide an embedded structure of the global noisy response behavior. The presence of light random perturbations, including tank noise and “designed” perturbations may cause variation in response amplitude and/or phase, and it may also lead to various transition behaviors among coexisting response attractors. A detailed global and local deterministic analysis could identify the embedded response stability structure, indicating possible transition routes and variations.

\section{Frequency Response Diagram}

Comparison of simulated responses and experimental results is shown in Fig.6 in Part I and good agreement is observed (see Part I for more detailed discussions). For interpretation purpose, the characteristic behavior and comparisons of the global experimental response behavior are briefly discussed here.

It is observed that the simulated responses match well the measured data in amplitude and characteristics over the frequency range examined. Primary resonance at near $0.26 \mathrm{~Hz}$ and secondary resonances at near 0.13 and $0.52 \mathrm{~Hz}$, respectively, are closely simulated. Coexisting 
harmonic and sub-harmonic responses near $0.5 \mathrm{~Hz}$ in the experimental results are also clearly demonstrated. Additionally, the simulated solutions indicate the potential existence of "verylarge-amplitude” harmonic responses in the frequency range of [0.25 0.53] Hz, which was not experimentally observed. The coexisting large-amplitude responses resulted from large initial conditions. Most of the experimental search runs started with the quiescent initial conditions, and the model response mostly follows the lower (smaller amplitude) response curve. The “very-large-amplitude” responses are out of the limit of the mechanical configuration designed

for the experiment and were not captured. However, for some tests at wave frequency near 0.27 $\mathrm{Hz}$, potential large motion was indicated. In those tests, the sphere was led to a very large amplitude such that the strings connecting the springs and sphere popped off the pulleys, and consequently the test was terminated. The tendency of reaching the "very-large-amplitude" motions may be resulted from a perturbation-induced transition. With the presence of perturbations consisted of tank noise and wave amplitude variations, the sphere was driven to the higher response curve (larger amplitude) that is beyond the limitation of the experimental configuration. Attempts of the response to reach the very-large-amplitude motions lead to the strings popping out of the pulleys.

\section{Resonance Regions}

Waves of all the periodic-with-noise tests were designed to be of 2 seconds (or $0.5 \mathrm{~Hz}$ ), which is near the sub-harmonic resonance. A local deterministic analysis is thus conducted with varied wave amplitude, and fixed period of 2 seconds to identify possible coexisting response attractors and associated transitions. Two additional numerical analyses are conducted near the regions of super-harmonic $(0.125 \mathrm{~Hz})$ and primary resonances $(0.25 \mathrm{~Hz})$ in attempt to further identify unexpected transitions in response characteristics (e.g., small- to large-amplitude harmonics, and harmonics to sub-harmonics).

Sub-harmonic Resonance -- Figure 1 shows a nonlinear relationship between the wave and response amplitudes. The wave amplitude varies from 0.02 to $2.2 \mathrm{ft}$, and the corresponding 
response amplitude various from 0.01 to $3.8 \mathrm{ft}$. Two response amplitude curves are noted. When the wave amplitude is lower than $0.75 \mathrm{ft}$, the (lower) response curve indicates a unique existence of small harmonic response whose amplitude increases with the wave amplitude. A sample small-amplitude harmonic response is shown in Fig.2a. A stability bifurcation is noted at wave amplitude at $0.7 \mathrm{ft}$, where the small amplitude harmonics disappear and sub-harmonics become dominant. The sub-harmonics were mostly observed in the experiment with 2-second waves. A sample sub-harmonic response is then shown in Fig.2b. When the wave amplitude is higher than $1.5 \mathrm{ft}$, the sub-harmonic responses disappear, and small amplitude harmonics reappear with amplitude increasing with the wave amplitude. It is also observed that there coexists a large-amplitude harmonic response (a sample as shown in Fig.2c) when the wave amplitude is higher than $0.8 \mathrm{ft}$.

Primary Resonance -- Figure 3 shows another series of investigation on the relationship at wave frequency of $0.27 \mathrm{~Hz}$. The wave amplitude varies from 0.02 to $2.2 \mathrm{ft}$, and the corresponding response amplitude varies from 0.01 to $3.0 \mathrm{ft}$. Two distinct response amplitude curves are noted. Small-amplitude harmonic response exists when the wave amplitude is with the range of [0 0.3]. A sample small-amplitude harmonic response is shown in Fig.4a. Largeamplitude responses occur when the wave amplitude increases to and beyond $0.2 \mathrm{ft}$. When the wave amplitude is in the range of [0.2 0.3] ft, small- and large- amplitude harmonic responses are indicated to coexist with various initial conditions. When the wave amplitude increases beyond $0.3 \mathrm{ft}$, the structural response is dominated by large-amplitude harmonics (a sample as shown in Fig.4b). As discussed in the previous section, the coexisting large amplitude harmonic response may be the underlying cause for experimentally observed "out-of-limit” motions near $0.27 \mathrm{~Hz}$.

Super-harmonic Resonance -- Figure 5 shows the investigation at wave frequency of 0.13 Hz. The wave amplitude varies from 0.2 to $2.2 \mathrm{ft}$, and the corresponding response amplitude varies from 0.25 to $1.65 \mathrm{ft}$. There only exists one continuous response amplitude curve in this case. The structure behaves mostly in super-harmonic fashion. However, a turning point of the 
curve at near wave amplitude of $1.3 \mathrm{ft}$ indicates that although still super-harmonic, transitions in response amplitude and characteristics occur. Sample super-harmonic responses in each stability region are shown in Fig.6.

The results of the analysis provide a framework for interpretations of noise effects on transitions in nonlinear response characteristics. It is implied that with the presence of random perturbations, the response trajectory may travel between the coexisting response attractors, namely, sub-harmonics, small- and large- amplitude harmonics. Comparisons with experimental results and interpretations will be elaborated in a later section.

\section{Comparisons and Discussions}

A detailed analysis of the data on the noise-induced inter-domain transitions and the noise effects on the response characteristics and system performance is shown in the following sections.

\section{Transition Behaviors}

As mentioned, besides the designed additive random perturbations, there is weak uncontrollable tank noise present in the wave excitation. With the tank noise presence, there exist unexpected transition behavior even in a "designed deterministic setting” from a steadystate response to another coexisting, yet distinct, response. When the noise intensity increases with the addition of designed random perturbations, interactions between coexisting responses could become more apparent. The observed transitions of coexisting responses are examined in detail by comparing with their corresponding deterministic counterparts. The interactions of coexisting responses are also examined by comparing with simulations and interpreted based on the embedded structure exhibited in the deterministic analysis.

The time history of an experimental “designed” deterministic response (Test D1) with system parameters and excitation details in the stability region of a single attractor is shown in Fig. 7a. Small variations in the response amplitude caused by the weak tank noise are noted to 
oscillate along its deterministic counterpart. As shown in the deterministic analysis, there exists a single response attractor. Figure 7b shows a time history of a "designed" deterministic model response (Test D2) with system parameters and excitation details in the stability region of multiple coexisting attractors. The response is observed to stay in a harmonic fashion for about 120 seconds, and then to transition to a sub-harmonic mode. The deterministic analysis predicts that there coexist two distinct response attractors, harmonic and sub-harmonic. Because of the variation in the wave amplitude (from 0.8 to $1.2 \mathrm{ft}$ ), the response trajectory accordingly drifts to and from the two attractors (cf. Fig.1).

The time histories of model responses with two-coexisting attractors to random perturbations with moderate intensities are shown in Fig. 8. It is observed that the response attractors are well-bridged due to the presence of designed noise perturbations. The response trajectory oscillates between harmonic and sub-harmonic modes as shown in Fig.8a. The transition phenomenon becomes more pronounced as the noise intensity increases. The wave amplitude varies between 0.2 and $1.0 \mathrm{ft}$, and the response trajectory rifts between harmonic and sub-harmonic response attractors as indicated in Fig.1. When the noise intensity becomes large (Fig.8b), the corresponding response time history becomes more random-like. The individual harmonic and sub-harmonic attractors can barely be traced.

\section{Chaotic-Like Response Behavior}

As demonstrated and discussed by Yim and Lin [8], there exists a seemingly complex experimental response time. By analyzing the response on the Poincaré section and its associated Poincaré time history, it is indicated that the response may be a result of noise-bridged multiple coexisting responses (four in this case) or noisy chaos. However, more detailed studies on classifying its characteristics are also strongly suggested [8].

The experimental responses are sampled and examined on a Poincaré section. A Poincaré point is sampled at time interval of forcing excitation period to suppress periodicity due to the dominant periodic excitation component. A Poincaré map is formed by a collection of 
Poincaré points. Concentration of Poincaré points on the section indicates the corresponding attractors.

Figure 9 shows the wave profile of the test, which indicates that the dominant frequency is at $0.5 \mathrm{~Hz}$ with wave amplitude varying within 0.8 and $1.5 \mathrm{ft}$. By comparing with the response curve obtained from the deterministic analysis (Fig.3), it is implied that there may coexist smalland large -amplitude harmonic attractors, but exist no possible chaos or more-than-two multiple attractors. Also, based on the numerical simulations, the initial conditions have to be large (e.g., $(-3 \mathrm{ft}, 3 \mathrm{ft} / \mathrm{s})$ ) to reach the large-amplitude harmonic domain. Note that in the wave profile, there are transitions among various wave trains, which commonly occur in long random wave trains due to facility limitations. The transitions in wave profile might cause corresponding phase shifts, and numerical simulations random perturbations in wave amplitude with uniformly distributed random phases are accordingly conducted to assimilate the experimental result.

The Poincaré map of a sample experimental model response (Test D13) is shown in Fig. 10a. The scattering of the points implies either noise-bridged multiple coexisting response attractors or a single noisy chaotic attractor. In this case, a Poincaré time history of the response (Poincaré points vs. time) may better reveal the response characteristics. It is shown that there are transitions among different steady states. Specifically, there are seven transitions occurring at around the $300^{\text {th }}, 425^{\text {th }}, 550^{\text {th }}, 1,175^{\text {th }}, 1,450^{\text {th }}, 1,575^{\text {th }}$ and $1,700^{\text {th }}$ second, respectively (not shown here due to page limit). The Poincaré time history is accordingly divided into 8 segments. One single attractor is assumed to embed in each response segment, and transitions from one attractor to another are attributed to the presence of noise. It is noted that one of the embedded attractors shows structured spreading that resembles the shape of the full-length data (cf. Fig.8 in [8]), and is suspected to be noisy chaotic. Spectral analysis and Lyapounov exponent calculation have been applied to these attractors in an attempt to quantitatively identify noisy chaos. However, due to the fact that the response is perturbed by a relatively strong noise, no definite conclusions can be made with quantitative evidences.

Figure10a shows the experimental result sampled on Poincaré section and compared to 
numerical simulation (Fig.10b). Good agreement in characteristics and shape is observed. Therefore, the strange-attractor like shape might be a combination of effects due to variations in wave amplitude and random phase. It is noted that near the region of $1 \mathrm{ft}$ wave amplitude, long transient periods (varying from 500 to 700 cycles of excitation period) in simulations are observed. The long transient periods may also contribute appearing complexity in the corresponding experimental result.

\section{Concluding Remarks}

This study presents detailed comparisons of numerical predictions and existing experimental results of a nonlinear, moored, submerged structural system subjected to nearly periodic wave excitations. Based on the results presented, the concluding remarks are summarized in order as follows:

1. Good agreement of IFF model predictions with the experimental results indicates the validity of the model. The model predictions are in good agreement with experimental results in both time and frequency domains.

2 Noise effects on the model results are examined and interpreted based on the corresponding detailed deterministic analysis. Results from the deterministic analysis depict all possible, multiple coexisting nonlinear responses. Possible transitions and interactions of these attractors are also indicated. Numerical results show that near the primary resonance, small-amplitude harmonics coexists with large-amplitude harmonics. It is also shown that there exists only a single response attractor near the super-harmonic resonance, and that there coexist sub-harmonics and large-amplitude harmonics near the sub-harmonic resonance.

3 Possible noisy chaotic response is further examined here in detail for its classification. Results of the corresponding deterministic analysis indicate neither chaotic response nor multiple response attractors existing in the specified parameter space. The noisy-chaotic like response behavior are possibly caused by transitions (varied phase shifts) in the 
waves. Simulations accordingly conducted show a good agreement to the experimental result in characteristics and amplitude.

\section{Acknowledgments}

Financial support from the United States Office of Naval Research Grant No. N0001492-J-1221 is gratefully acknowledged.

\section{References}

[1] Gottlieb, O., and Yim, S.C.S., 1992, "Nonlinear oscillations, bifurcations and chaos in a multi-point mooring system with a geometric nonlinearity,” Appl. Ocean Res., 14, pp. 241-257.

[2] Gottlieb, O., and Yim, S.C.S., 1993, "Drag-induced instability and chaos in mooring systems," Ocean Engng., 29, pp. 569-599.

[3] Gottlieb, O., Yim, S.C.S., and Lin H., 1997, “Analysis of bifurcation superstructure nonlinear ocean system,” J. Engng. Mech. ASCE, 123, pp. 1180-1187.

[4] Isaacson, M., and Phadke, A., 1994, "Chaotic motion of a nonlinearly moored structure," Proc. $4^{\text {th }}$ Intl. Offshore and Polar Engineering Conference, III, pp. 338-345.

[5] Yim, S.C.S., Myrum, M.A., Gottlieb, O., Lin, H., and Shih, I-Ming, 1993, Summary and Preliminary Analysis of Nonlinear Oscillations in a Submerged Mooring System Experiment, Report No. OE-93-03, Ocean Engineering Program, Oregon State University.

[6] Lin, H., and Yim, S.C.S., 1997, “Noisy nonlinear motions of a moored system, Part I: analysis and simulations,” J. Wtrwy., Port, Coast. and Oc. Engng. ASCE, 123, pp. 287295.

[7] Lin, H., Yim, S.C.S., and Gottlieb O., 1998, "Experimental investigation in bifurcations of an ocean system,” Ocean Engng., 25 4/5, pp. 323-343.

[8] Yim, S.C.S., and Lin, H., 2000, "Noisy nonlinear motions of a moored system, Part II: 
experimental study,” J. Wtrwy., Port, Coast. and Oc. Engng. ASCE, 126, pp. 113-120. 


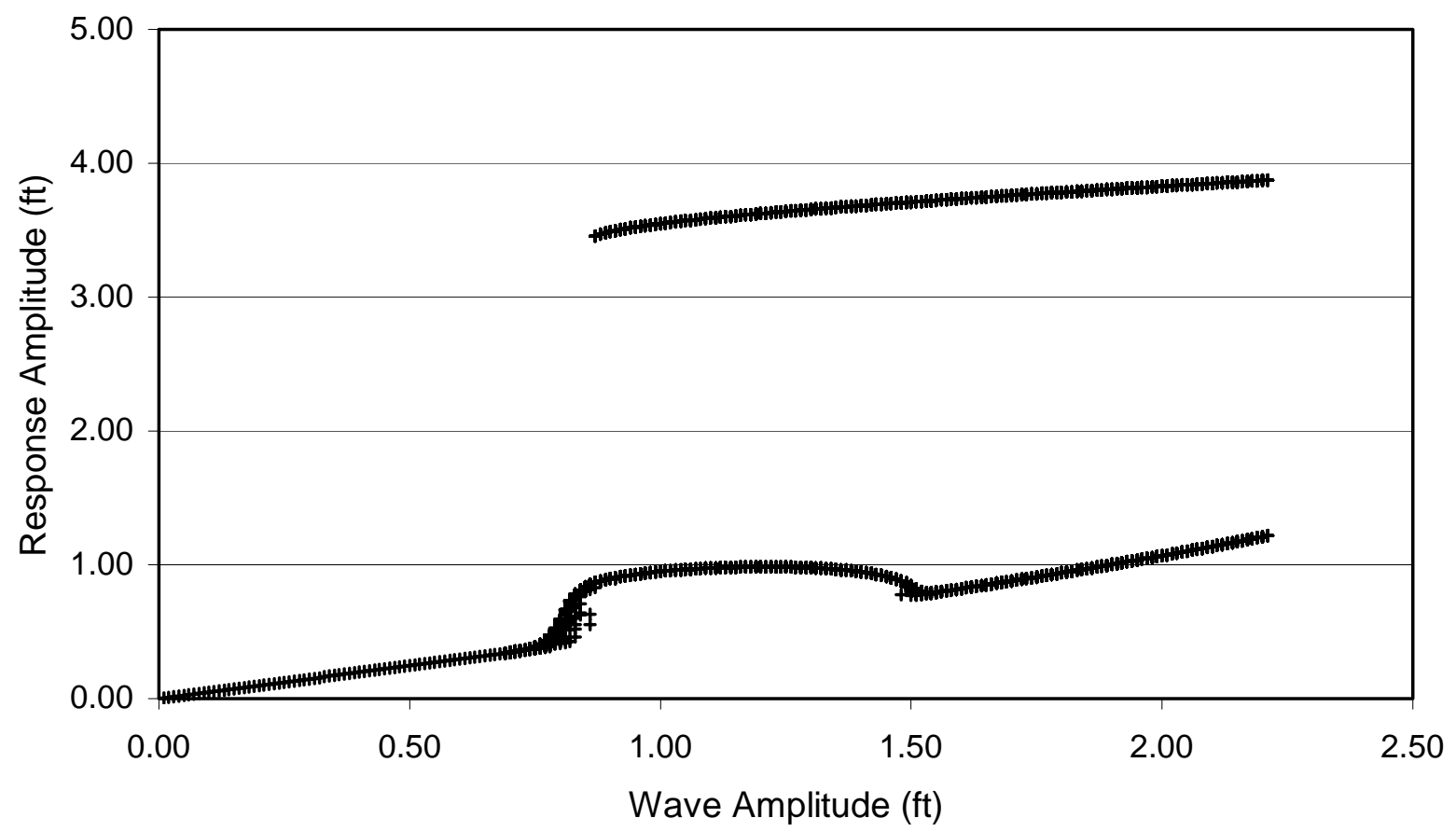

Fig. 1 Amplitude response curve near sub-harmonic resonance $(0.5 \mathrm{~Hz}) ; C_{A}=0.25, C_{D}=0.1$, $k_{1}=9.3(\mathrm{lb} / \mathrm{ft}), k_{2}=4.0\left(\mathrm{lb} / \mathrm{ft}^{2}\right), k_{3}=4.0\left(1 \mathrm{~b} / \mathrm{ft}^{3}\right), C_{D}^{\prime}=0.02$, and $\zeta_{S}=6 \%$

Lin, Huan Journal of OMAE 
a)

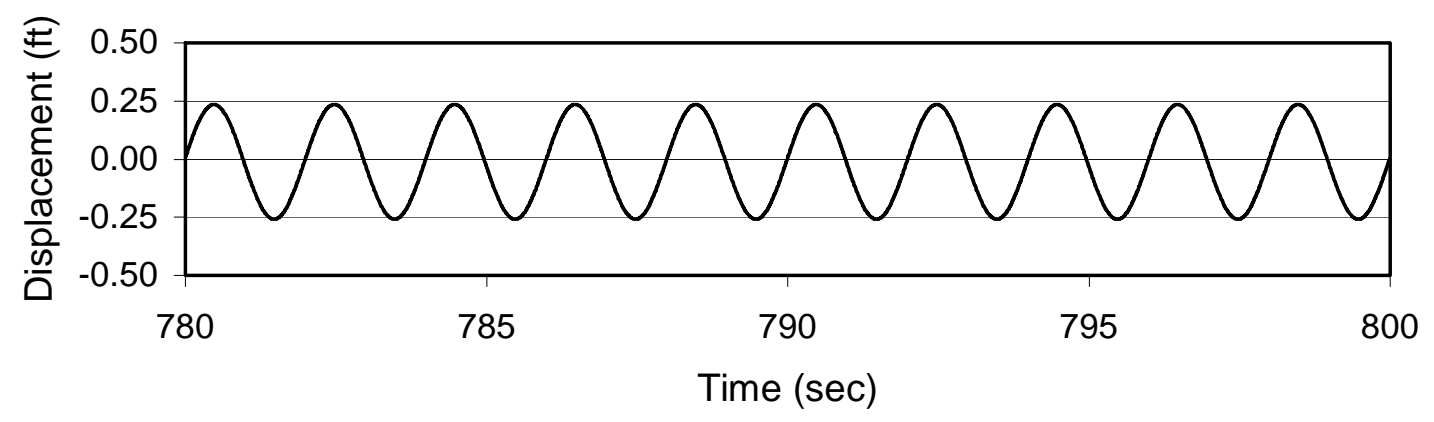

b)

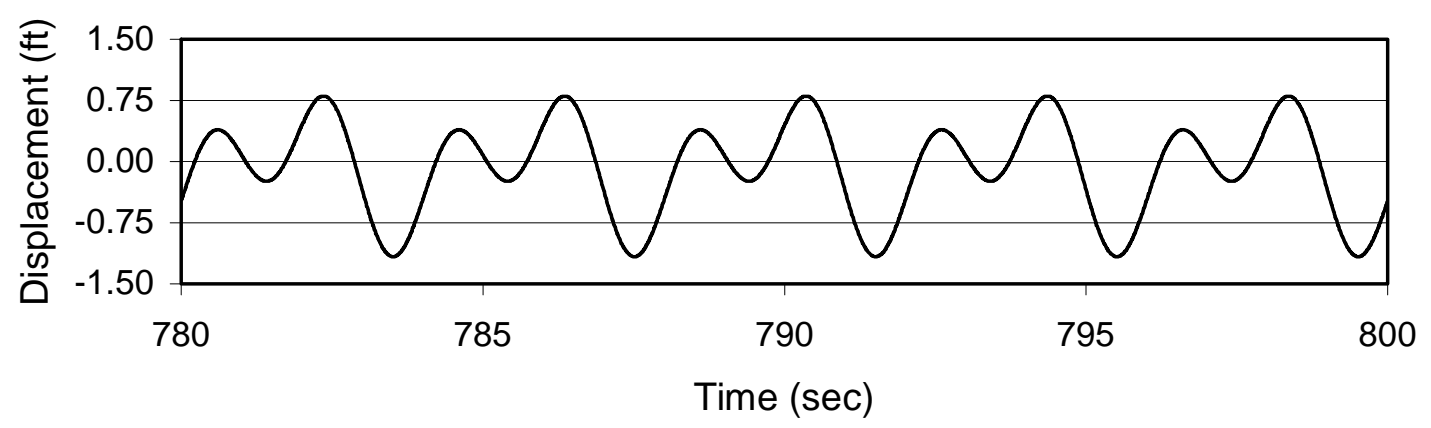

c)

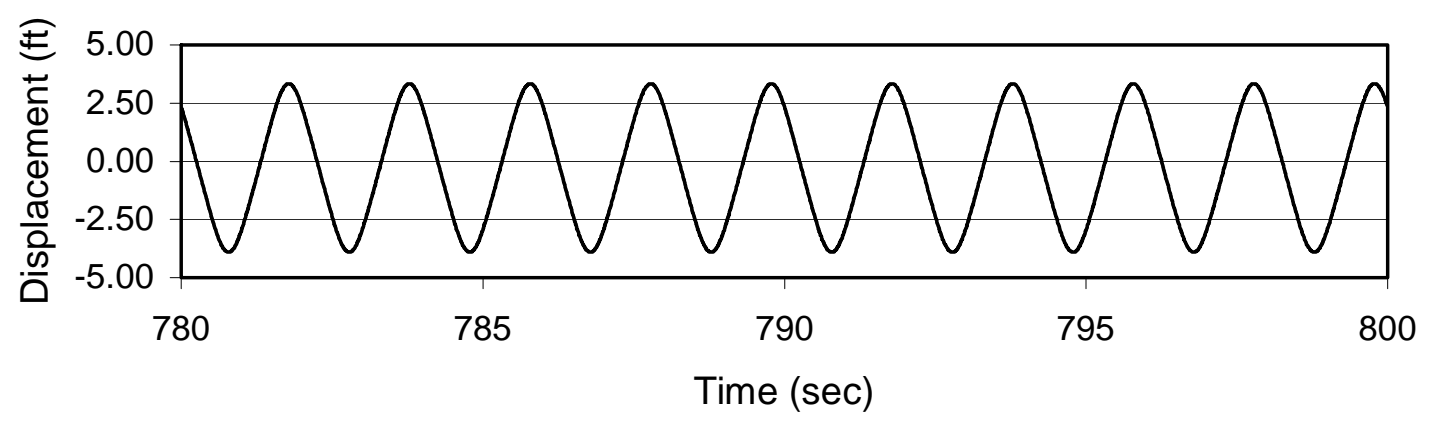

Fig. 2 Time histories of sample responses near sub-harmonic resonance $(0.5 \mathrm{~Hz})$ : a) smallamplitude harmonics (wave amplitude of $0.5 \mathrm{ft}$ ), b) sub-harmonics, and c) largeamplitude harmonics (wave amplitude of $0.8 \mathrm{ft}$ )

Lin, Huan

Journal of OMAE 


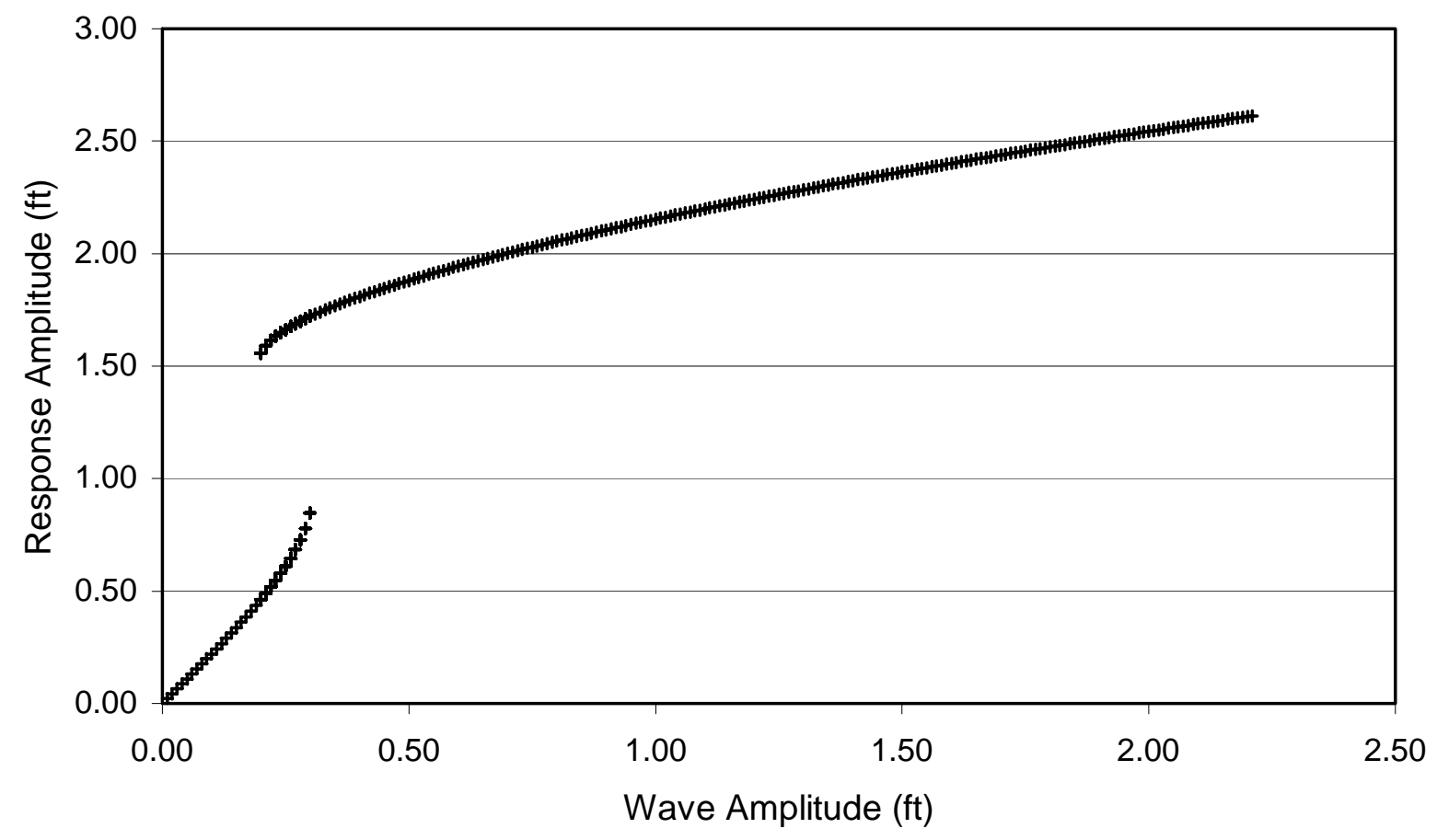

Fig. 3 Amplitude response curve near primary resonance $(0.27 \mathrm{~Hz}) ; C_{A}=0.25, C_{D}=0.1$, $k_{1}=9.3(\mathrm{lb} / \mathrm{ft}), k_{2}=4.0\left(\mathrm{lb} / \mathrm{ft}^{2}\right), k_{3}=4.0\left(1 \mathrm{~b} / \mathrm{ft}^{3}\right), C_{D}^{\prime}=0.02$, and $\zeta_{S}=6 \%$ 
a)

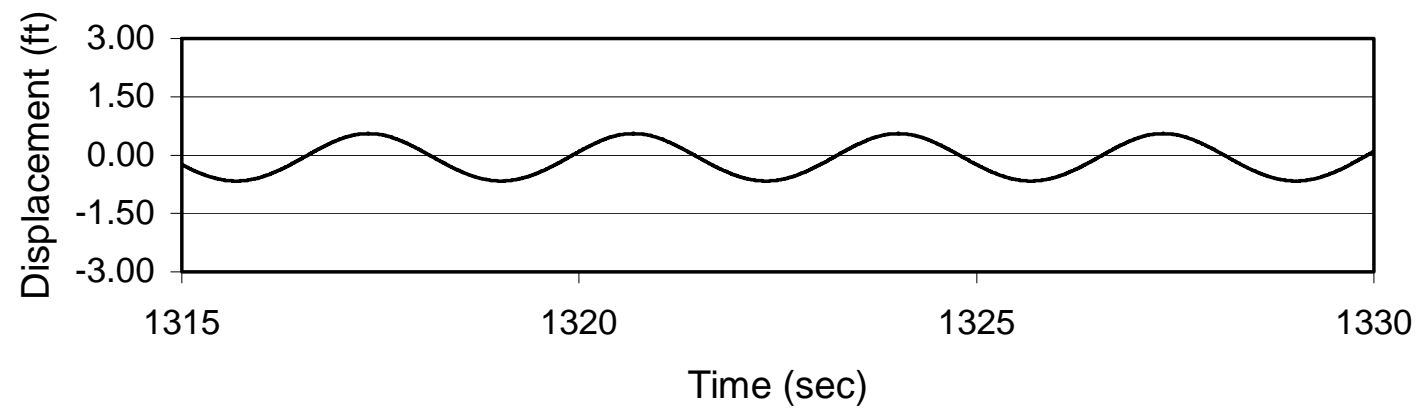

b)

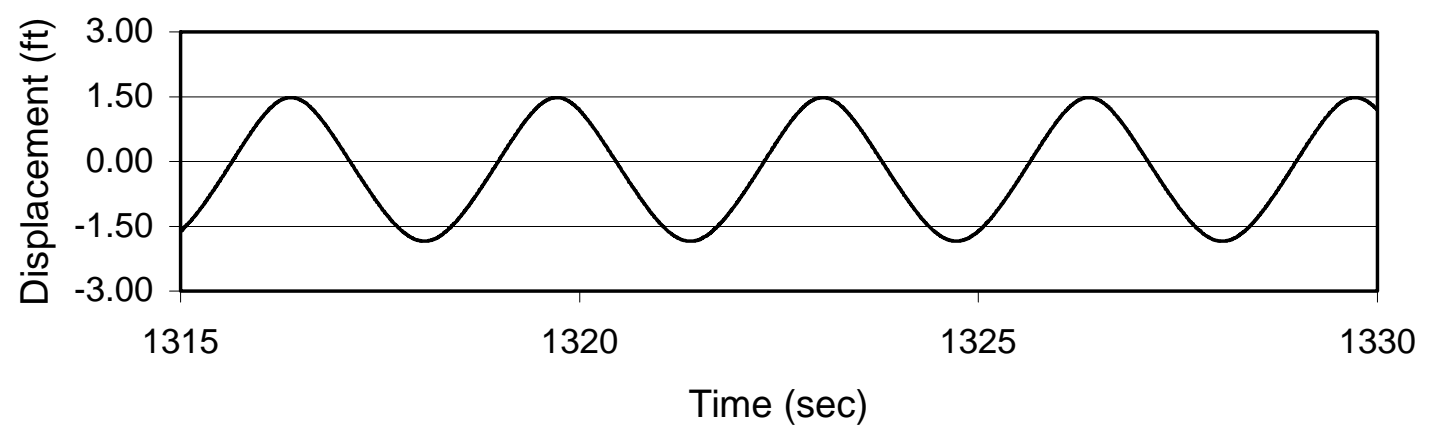

Fig. 4 Coexisting harmonic response with wave amplitude of $0.25 \mathrm{ft}$ near primary resonance $(0.27 \mathrm{~Hz})$; a) small amplitude, and b) large amplitude

Lin, Huan Journal of OMAE 


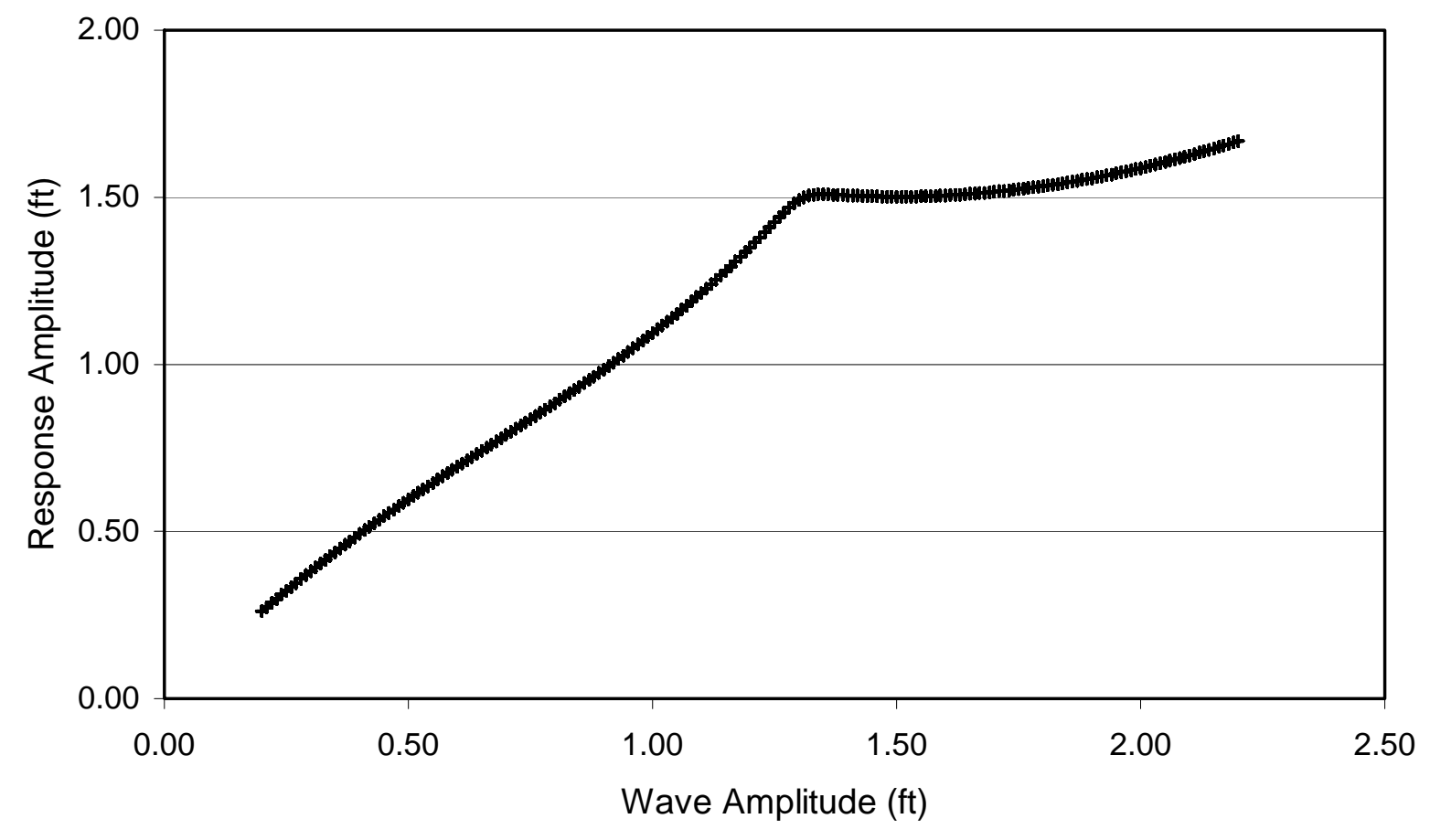

Fig. 5 Amplitude response curve near super-harmonic resonance $(0.125 \mathrm{~Hz}) ; C_{A}=0.25, C_{D}=0.1$, $k_{1}=9.3(\mathrm{lb} / \mathrm{ft}), k_{2}=4.0\left(\mathrm{lb} / \mathrm{ft}^{2}\right), k_{3}=4.0\left(1 \mathrm{~b} / \mathrm{ft}^{3}\right), C_{D}^{\prime}=0.02$, and $\zeta_{S}=6 \%$

Lin, Huan Journal of OMAE 
a)

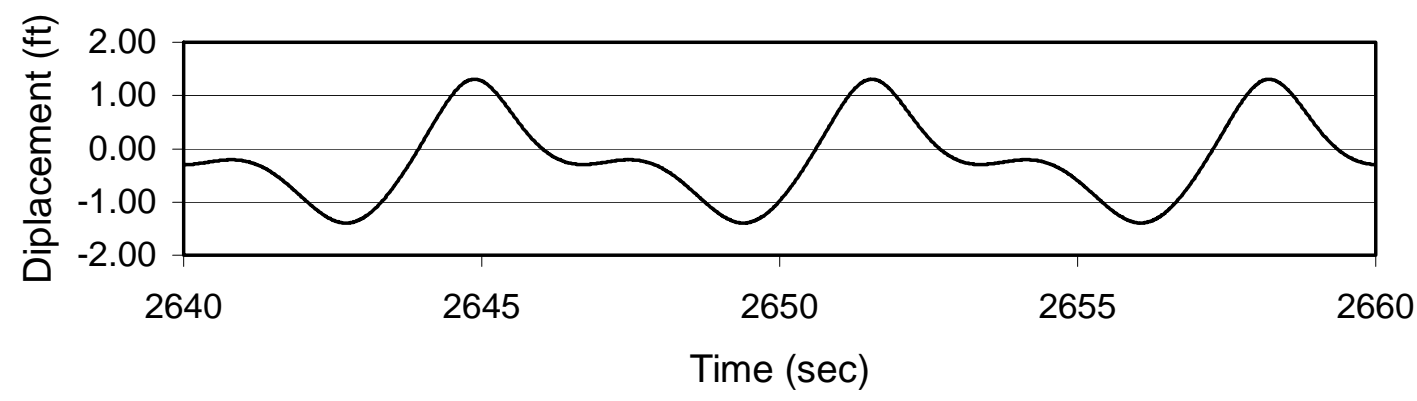

b)

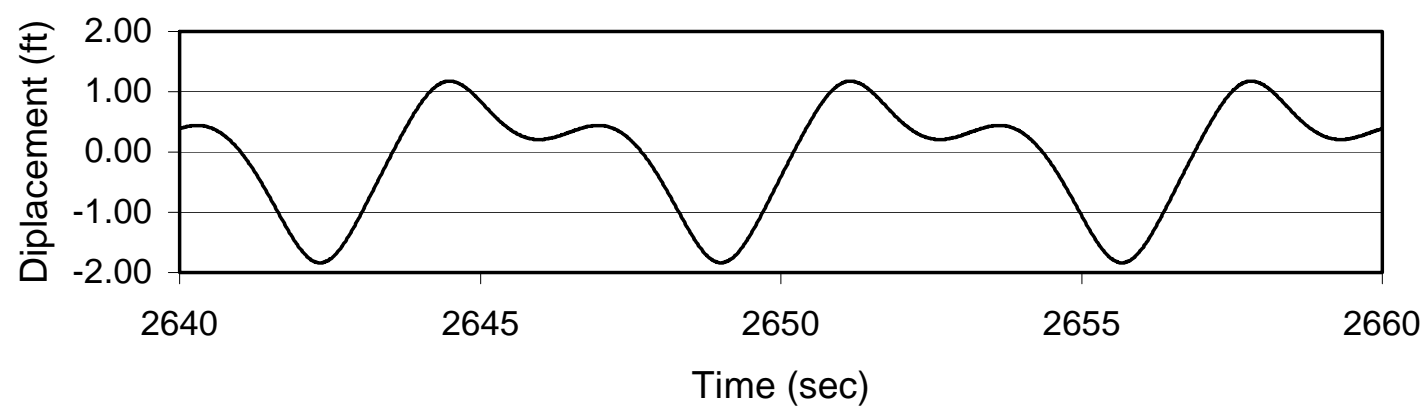

Fig. 6 Super-harmonics near super-harmonic resonance $(0.125 \mathrm{~Hz})$ with wave amplitude $=$ a) $1.25 \mathrm{ft}$, and b) $1.35 \mathrm{ft}$

Lin, Huan Journal of OMAE 
a)

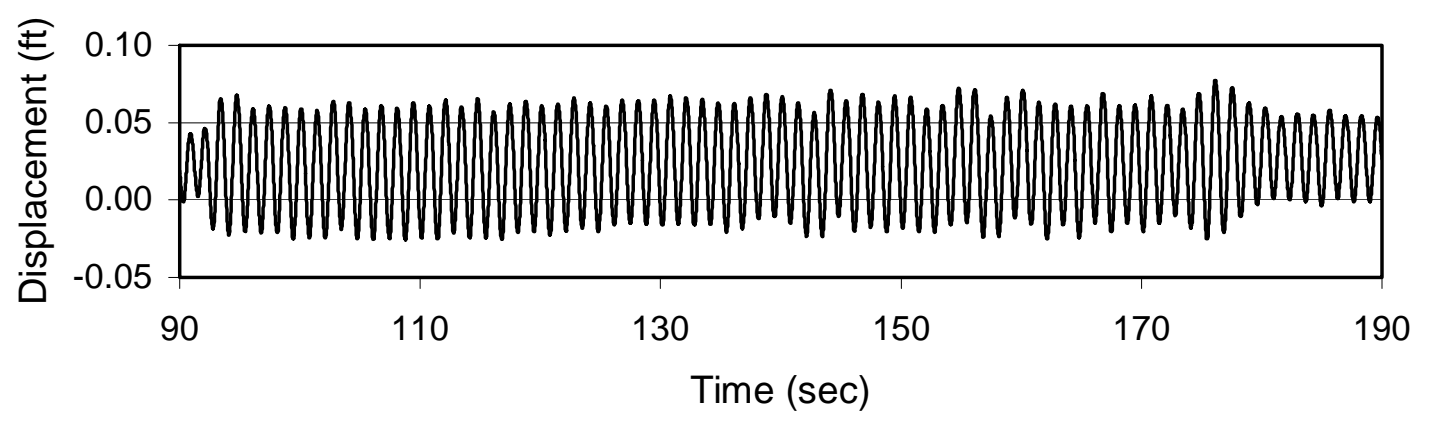

b)

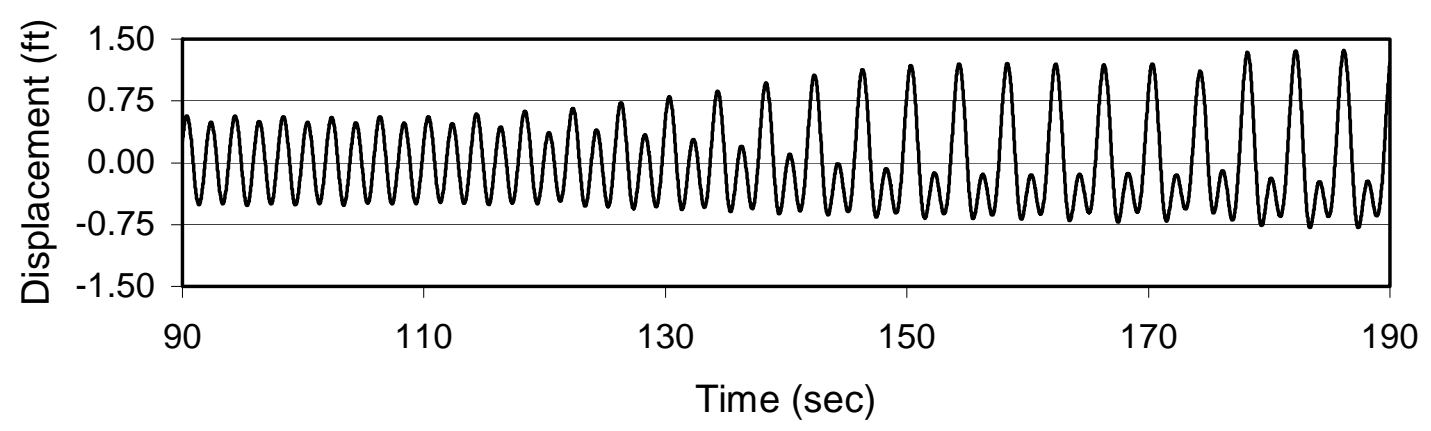

Fig. 7 Noise-induced transitions on "designed” deterministic experimental responses:

a) single response attractor (Test D1), and b) coexisting harmonic and sub-

b) harmonic responses (Test D2)

Lin, Huan

Journal of OMAE 
a)

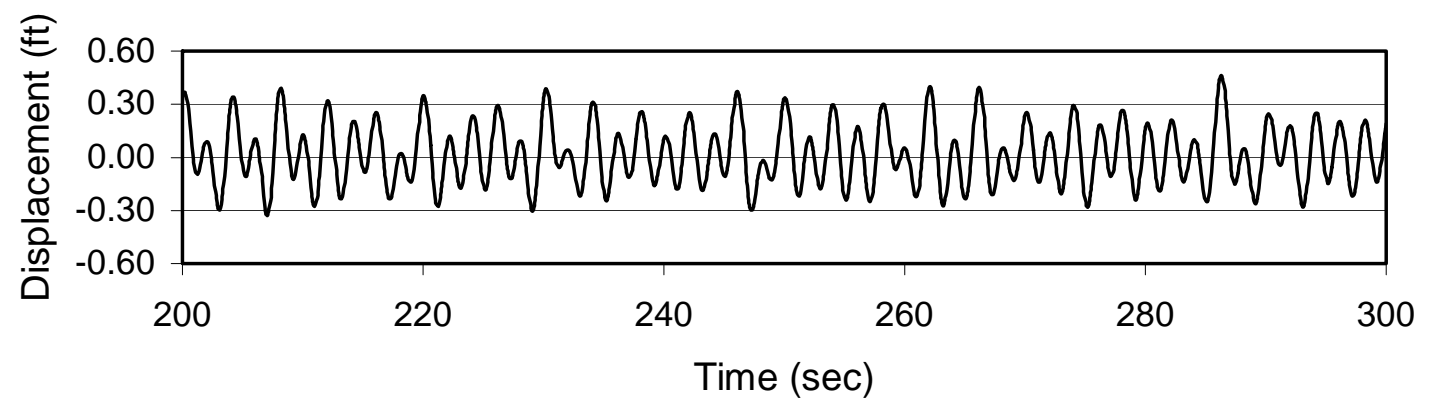

b)

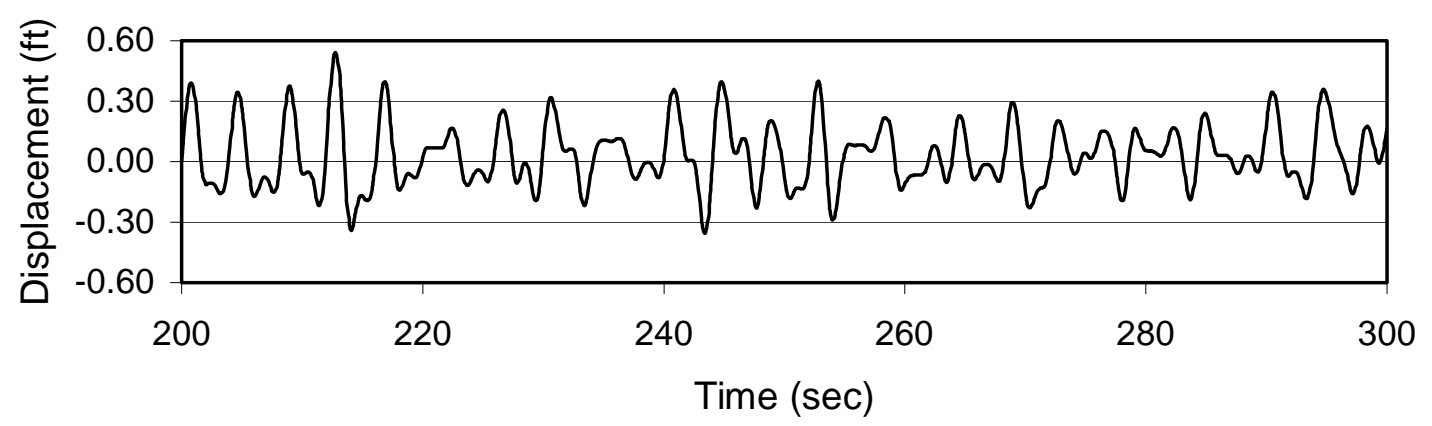

Fig. 8 Noisy experimental responses: a) small noise intensity with variance $\sim 0.01$ (Test D7), and b) larger noise intensity with variance $\sim 0.04$ (Test D6)

Lin, Huan

Journal of OMAE 
a)

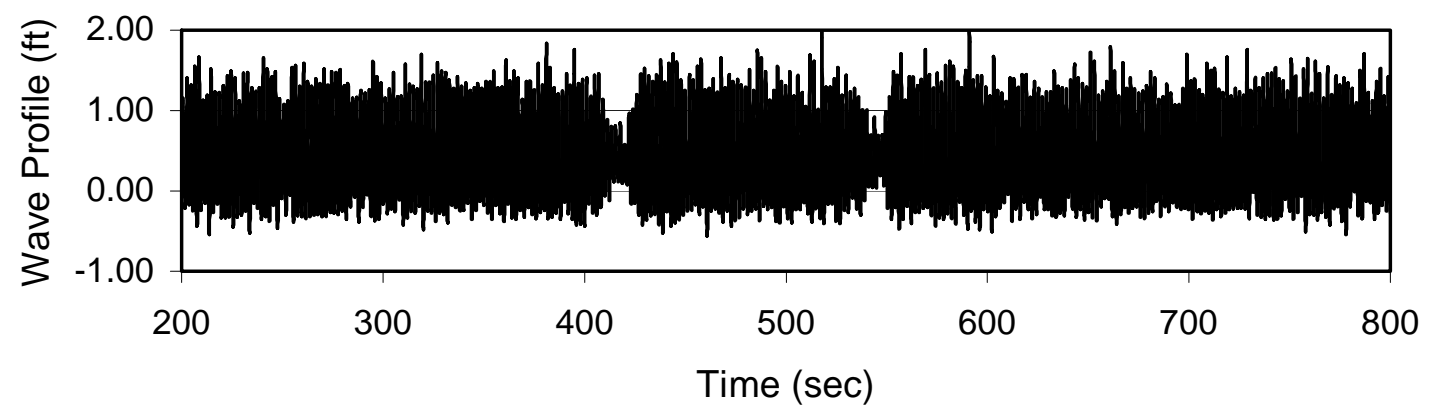

b)

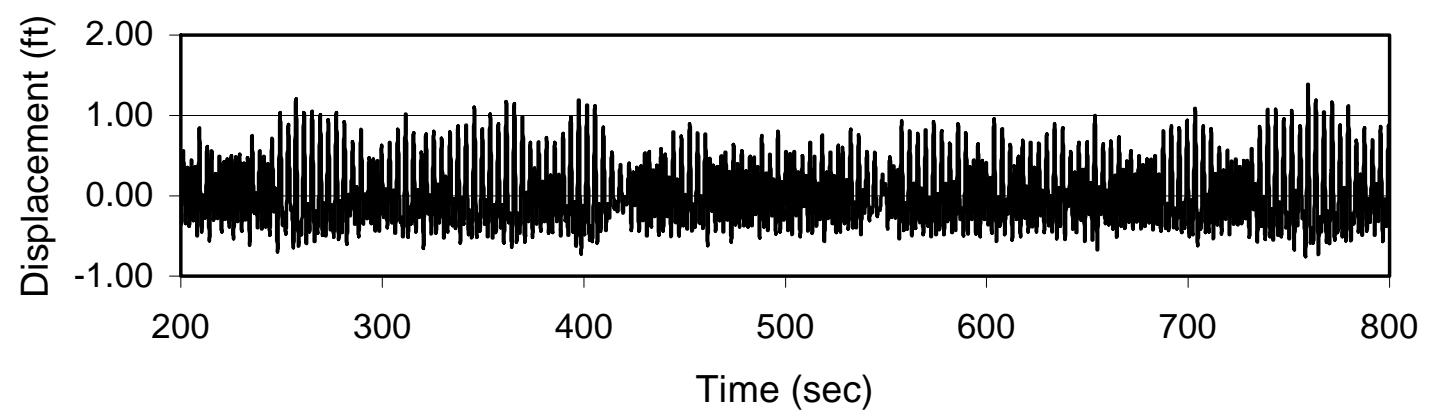

Fig. 9 Possible noisy-chaotic experimental response at wave frequency of $0.5 \mathrm{~Hz}$ (Test D13): a) wave profile, and b) response 
a)

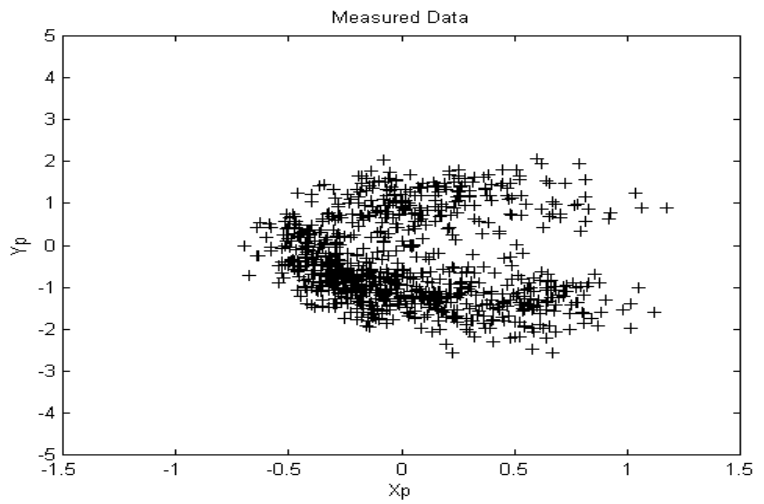

b)

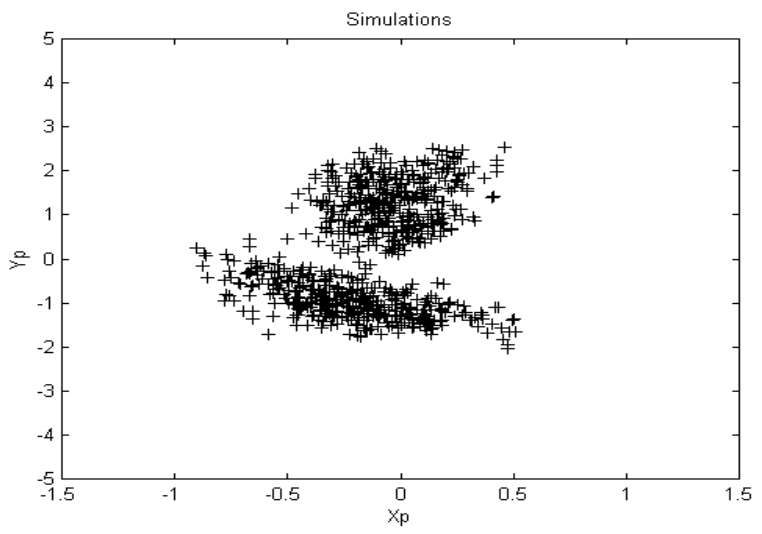

Fig. 10 "Possible" noisy chaotic response on Poincaré map: a) measured data, and b) simulations $\left(C_{A}=0.25, C_{D}=0.1, k_{1}=9.3(\mathrm{lb} / \mathrm{ft}), k_{2}=4.0\left(\mathrm{lb} / \mathrm{ft}^{2}\right), k_{3}=4.0\left(1 \mathrm{~b} / \mathrm{ft}^{3}\right)\right.$, $C_{D}^{\prime}=0.02$, and $\zeta_{S}=6 \%$ ) 